\title{
Perceptions of tuberculosis and treatment seeking behaviour in Ilala and Kinondoni Municipalities in Tanzania
}

\author{
A.M. KILALE ${ }^{1 *}$, A.K. MUSHI ${ }^{2}$, L.A. LEMA ${ }^{1}$, J. KUNDA ${ }^{1}$, C.E. MAKASI ${ }^{1}$, D. MWASEBA ${ }^{1}$, \\ N.S. RANGE ${ }^{1}$ and G.S. MFINANGA ${ }^{1}$ \\ ${ }^{1}$ National Institute for Medical Research, Muhimbili Medical Research Centre, P.O. Box 3436, Dar es Salaam, \\ Tanzania \\ ${ }^{2}$ National Institute for Medical Research, Amani Medical Research Centre, Muheza, Tanzania
}

\begin{abstract}
This study was carried out in Ilala and Kinondoni Municipalities in Tanzania to explore the perceptions of Tuberculosis (TB), and treatment seeking behaviour, among patients attending healthcare facilities. The study was conducted in four randomly selected health facilities providing directly observed treatment (DOT). Exit interviews were administered to 69 randomly selected TB patients. The mean age of the respondents was 33.2 years (range $=11-72$ years). Forty-six $(66.7 \%)$ of the patients had primary school education. Fifty-nine $(84.1 \%)$ patients had good knowledge on the transmission of TB. Majority $(75 \%)$ of the respondents were of the opinion that the incidence of TB was on the increase and this was mainly associated with HIV/AIDS epidemic. All respondents knew that TB was a curable disease if one complies with the treatment. Sixty-four $(60 \%)$ respondents had good knowledge on the correct duration of tuberculosis treatment. The median duration before seeking treatment from a health facility was 1.5 months. The majority of the patients $47(68 \%)$ visited public health facilities for treatment as their first action. Overall, 83.8\% (57/68) respondents said females comply better with treatment than male patients. The majority of the respondents lived within a walking distance to a healthcare facility. Only $18.8 \%(13 / 69)$ had to spend an average of US\$ $0.2-0.3$ as travel costs to the healthcare facility. Most of the respondents $(57.8 \%)$ said they were well attended by service providers. Half (21/42) and 59.3\% (16/27) of the males and females, respectively, mentioned good patient-service provider relationship as an important reason for satisfaction of the service $\left(\chi^{2}=0.57, \mathrm{df}=1, P>0.005\right)$. Twenty-nine ( $\left.42 \%\right)$ of respondents were of the opinion that female TB patients conformed better to treatment than males and a similar number thought that both of them equally conformed to treatment. Findings from this study indicate that a large population in urban settings are aware that health facilities play a major role in TB treatment. In conclusion, there is a need to further explore how this information could potentially be used to enhance early seeking of appropriate services among TB patients in the era of rapid urbanization. Strategies in the control of TB and other diseases should focus on advocacy in seeking appropriate care.
\end{abstract}

Key words: tuberculosis, perceptions, gender, treatment, behaviour, Tanzania

\section{Introduction}

Globally, tuberculosis (TB) kills approximately 5000 people daily, with $98 \%$ of deaths occurring in developing world, affecting mostly young adults in their most productive years (WHO, 2005). In recent years, TB has been reported to be the leading killer among HIV-infected people with weakened immune systems; and that a quarter of a million TB deaths are HIV-associated, with most of them in Africa (WHO, 2005). The Direct Observed Treatment Short course (DOTS) is the main strategy in the control of tuberculosis. The strategy launched in 1995, includes diagnosis through bacteriology and standardised short-course chemotherapy with full patient support (WHO, 2002).

Despite the effectiveness of the DOTS strategy, delays in seeking care among tuberculosis patients are common in a number of developing countries, including Tanzania (Zhang et al., 2007; Okeibunor et al., 2007; Irani et al., 2007). It has been reported that a reasonable number of tuberculosis patients are first treated by traditional medical practitioners before they seek care at healthcare facilities (Yamasaki-Nakagawa, 2001; Okeke \& Aguwa, 2006; Mangesho et al., 2007; Zhang et al., 2007). Delays in seeking care for TB have also been associated with stigma, in parts where the disease is considered as a 'dirty' disease, which mainly affects poor people.

Gender differences in seeking care has been reported in some countries. In Pakistan, women with respiratory symptoms had less access to public outpatient clinic than men and were unlikely to undergo sputum smear examination (Begum et al., 2001). Women with TB in Nepal were reported to delay in seeking care from health facilities as they opted to visit traditional healers before diagnosis than men (Yamasaki-Nakagawa, 2001). Studies conducted in Vietnam showed that women with pulmonary TB were diagnosed on average two weeks later than men due to delays caused by health care provider (Thorson \& Diwani, 2001). In Vietnam again, gender differences in health seeking behaviour were associated with fear of social isolation, economic constraints and poor quality of health services (Johansson et al., 2000). 
Understanding community treatment seeking behaviour and factors influencing the choice of healthcare is particularly important for evidence-based tuberculosis interventions. Such evidence is needed to devise properly targeted intervention strategies. Ilala and Kinondoni are two of the three municipalities that form the Dar es Salaam City in Tanzania. This study area was selected due to its typical example of the rapid urbanization process in sub-Saharan Africa. Health problems in the municipalities reflect the epidemiologic transition, with the emergence of chronic diseases, as well as the prevailing problems related to infectious diseases (Lorenz \& Mtasiwa, 2004). This study therefore, aimed to explore perceptions of TB and treatment seeking behaviour among urban populations of Tanzania. In addition, with a view to address issues related to social cost, the study explored and compared the experiences and expectations of male and female TB patients in seeking TB treatment in Ilala and Kinondoni, Tanzania.

\section{Materials and methods}

\section{Study site and data collections}

The study was conducted in four selected health facilities in urban and peri urban settings of Ilala and Kinondoni Municipalities in Dar es Salaam City, Tanzania. The facilities were randomly selected from a list of 16 hospitals, health centres and dispensaries providing TB DOTS services. The facilities included Mburahati and Mbezi dispensaries in Kinondoni and Vingunguti and Chanika in Ilala. At each health facility, exit interviews were administered to patients receiving TB treatment. In addition to their demographic information on age, education, marital status and occupation, the respondents were asked about income, distance to health facilities, cost of travel, social support and perceived social status as TB patients.

\section{Ethical issues}

The National Institute for Medical Research granted the ethical clearance to the study. Verbal consent was sought from all patients before they were enrolled into the study. Since the respondents were either interviewed at home or health facilities, consent was sought from either parent or relative for those who were aged below 15 years.

\section{Results}

\section{Demographic indices}

Sixty-nine TB patients were enrolled into the study (Table 1). Thirty-three $(47.8 \%)$ respondents were heads of the households, $29 \%$ (20/69) were spouses,
$17.4 \%(12 / 69)$ were sons or daughters (age $=11-20$ years) and $5.8 \%(4 / 69)$ were living with relatives. There were more males (61\%) than females $(\mathrm{P}<0.05)$. The mean age of the respondents was 33.2 years (range $=11-72$ years). Forty-six (66.7\%) of the patients had primary school education, eight (11.6\%) had never gone to school and nine (13\%) had secondary school education (Table 1. Eighteen (26.1\%) were business persons, 16 (23.2\%) housewives, $10(14.5 \%)$ farmers, $4(5.8 \%)$ drivers, $3(4.3 \%)$ and $2(2.9 \%)$ included a student and an artisan. Thirteen (46\%) of the respondents were on their fist two months of TB treatment, $31.9 \%(22 / 69)$ and $21.7 \%(15 / 69)$ were on the second and third months of TB treatment, respectively (Table 1).

\section{Knowledge on TB transmission}

Fifty-nine (84.1\%) patients had good knowledge on the transmission of TB. The males without knowledge on transmission of TB were $16.7 \%$ (7/42) and $14.8 \%$ $(4 / 27)$ were females $(P>0.05)$. Table 2 shows the responses of the TB patients on the knowledge about transmission of TB according to level of education.

Inhalation of air/dust containing TB infectious materials as major route of infection was mentioned by $49.1 \%(28 / 69)$ of the respondents. Infection from a coughing TB patient was reported by $19.3 \%(11 / 69)$ while other respondents associated the transmission of TB with congested accommodation and commuter buses, tongue kiss, sharing eating or drinking utensils with a TB patient, coming close to a TB patient, sharing meals or alcohol and sleeping with a TB patient.

\section{Perceptions of incidence of TB}

Majority (75\%) of the respondents were of the opinion that the incidence of TB was on the increase. However, eight $(13.3 \%)$ respondents thought the incidence was decreasing while two $(3.3 \%)$ said that it was normal. Five (8.4\%) respondents did not know anything on the magnitude of TB in their respective community. HIV/AIDS epidemic was associated with the increase in the incidence of TB. Other reasons included poor compliance to treatment, increase in population, overcrowding (in residential houses and commuter buses), negligence, ignorance, lack of isolation of TB patients, increased awareness about the disease among patients, heavy duties, and environmental pollution.

\section{Knowledge on TB treatment and cure}

All respondents knew that TB was a curable disease if one complies with the treatment, personal experience of improvement after starting treatment, availability of reliable drugs at health facilities and experience from other patients who had been cured and improvement 
Table 1: Demographic features of the study respondents

\begin{tabular}{lcl}
\hline \multicolumn{2}{l}{ Distribution of study patients by health facility } & \\
\hline Variable & Number of respondents & \\
\hline Age distribution of the patients (years) & 3 & 4.3 \\
$<15$ & 52 & 75.4 \\
$15-45$ & 14 & 20.6 \\
$>45$ & 69 & 100.0 \\
Total & & \\
\hline Level of education of the patients & 9 & 13.0 \\
Secondary School & 46 & 66.7 \\
Completed Primary Education & 6 & 8.7 \\
$<$ Standard 7 & 8 & 11.6 \\
Never gone to school & 69 & 100.0 \\
Total & 11 & 76.5 \\
\hline Distance from respondents' home to nearby health facility & 16.2 \\
$0-5$ km (< 1 hour walking) & 52 & 7.4 \\
$>5$ km (> 1 hour walking & 58 & 100 \\
Forced to use private transport & & \\
Total & 5 &
\end{tabular}

Table 2: Perception on tuberculosis and adherence to treatment

\begin{tabular}{|c|c|c|c|c|c|c|c|c|c|c|}
\hline \multicolumn{11}{|c|}{ a) Knowledge of transmission of tuberculosis } \\
\hline \multirow[t]{3}{*}{ Level of education } & \multicolumn{4}{|c|}{ Yes } & \multicolumn{4}{|c|}{ NO } & \multirow{2}{*}{\multicolumn{2}{|c|}{ Total }} \\
\hline & \multicolumn{2}{|c|}{ Males } & \multicolumn{2}{|c|}{ Females } & \multicolumn{2}{|c|}{ Males } & \multicolumn{2}{|c|}{ Females } & & \\
\hline & No. & $\%$ & & $\%$ & No. & $\%$ & No. & $\%$ & \multicolumn{2}{|c|}{ No. $\%$} \\
\hline Secondary School & 6 & 8.7 & 2 & 2.9 & 1 & 1.4 & 1 & 1.4 & 10 & 14.5 \\
\hline Completed Primary School & 26 & 37.7 & 16 & 23.2 & - & - & 4 & 5.8 & 46 & 66.7 \\
\hline$<$ standard Seven & 3 & 4.4 & 1 & 1.4 & 2 & 2.9 & - & - & 6 & 8.7 \\
\hline Never gone to school & 1 & 1.4 & 3 & 4.4 & 3 & 4.4 & - & - & 7 & 10.1 \\
\hline Total & 36 & 52.2 & 22 & 3.19 & 6 & 8.7 & 5 & 7.3 & 69 & 100 \\
\hline \multicolumn{11}{|c|}{ b) Opinion on who adheres well to TB treatment } \\
\hline \multirow[t]{2}{*}{ Opinion given by } & \multicolumn{2}{|c|}{ Males } & \multicolumn{2}{|c|}{ Females } & \multicolumn{2}{|c|}{ Equal } & \multicolumn{2}{|c|}{ Don't Know } & \multicolumn{2}{|c|}{ Total } \\
\hline & No. & $\%$ & No. & $\%$ & No. & $\%$ & No. & $\%$ & & \\
\hline Males & 4 & 9.5 & 16 & 38.1 & 17 & 40.5 & 5 & 11.9 & & 42 \\
\hline Females & - & - & 13 & 48.2 & 12 & 44.4 & 2 & 7.4 & & 27 \\
\hline Total & 4 & 5.8 & 29 & 42.0 & 29 & 420 & 7 & 102 & & 69 \\
\hline \multicolumn{11}{|c|}{ c) Perceived reasons for satisfaction } \\
\hline & \multicolumn{4}{|c|}{ Males } & \multicolumn{4}{|c|}{ Females } & \multicolumn{2}{|c|}{ Total } \\
\hline & \multicolumn{2}{|l|}{ No. } & \multicolumn{2}{|c|}{$\%$} & No. & & \multicolumn{2}{|l|}{$\%$} & \multicolumn{2}{|c|}{ No. $\%$} \\
\hline $\begin{array}{l}\text { Good patient-provider } \\
\text { relationship }\end{array}$ & \multicolumn{2}{|l|}{21} & \multicolumn{2}{|c|}{32.8} & \multicolumn{2}{|l|}{16} & \multicolumn{2}{|c|}{25.0} & 37 & 57.8 \\
\hline Reliable availability of drugs & \multicolumn{2}{|l|}{4} & \multicolumn{2}{|c|}{6.3} & - & & - & & 4 & 6.3 \\
\hline Time keeping/quick service & 4 & & 6.3 & & 2 & & 3.1 & & 6 & 9.4 \\
\hline Improvement from treatment & 5 & & 7.8 & & 3 & & 4.7 & & 8 & 12.5 \\
\hline Others & 5 & & 7.8 & & 4 & & 6.3 & & 9 & 14.1 \\
\hline Total & 39 & & 60. & & 25 & & 39 & & 64 & 100 \\
\hline
\end{tabular}


of those who were seriously sick.

Five $(7.2 \%)$ of the 69 respondents did not know the required duration to complete TB treatment. On the other hand, $64(60 \%)$ respondents reported the correct duration of 8 months and $35(50.7 \%)$ respondents mentioned the duration to be less or more than 8 months. About a half of the respondents reported to have taken 1- 4 weeks before seeking care from a health facility. Similarly, $23 \%$ and $24 \%$ of the respondents sought care from health facilities after 5-8 and 9-12 weeks, respectively. Some patients (3\%) sought treatment from health facilities after 3 months. The median duration before seeking treatment from a health facility was 1.5 months. More male respondents sought treatment from health facilities within the first 4-8 weeks than the

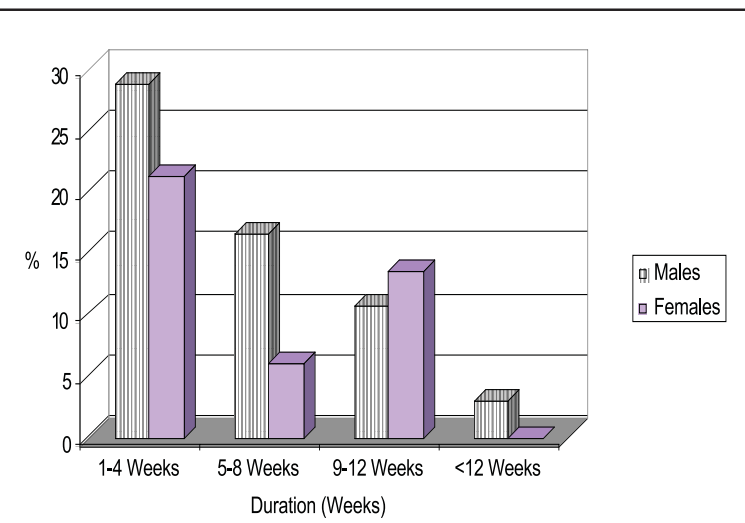

Figure 1: Duration of illness before presenting to health facility by sex females. However, the difference was not statistically significant (Figure 1) $(P>0.005)$.

\section{Choice or option for place of treatment}

The majority of the patients 47 (68\%) visited public health facilities for treatment of their illness as their first action. A few (3\%) went to traditional practitioners as their first action (Table 3). The majority of the respondents lived within a walking distance to a healthcare facility, thus encountered no cost for travelling to and from health facilities. Only 13\% (9/69) had to spend an average of US\$ 0.3 to and from DOTS centres and $17.4 \%(12 / 69)$ were spending US\$ 0.2 as travel costs for the same purpose.

\section{Service satisfaction and treatment compliance}

All interviewed patients were satisfied with the services provided to TB patients. Twenty one $(50 \%)$ of the 42 males mentioned good patient-service provider relationship as an important reason for satisfaction as compared to $59.3 \%(16 / 27)$ female respondents $\left(\chi^{2}\right.$ $=0.57$, df $=1, P>0.005)$ who gave the same reason. Most of the respondents $(57.8 \%(37 / 64))$ said they were well attended by service providers.

Twenty-nine $(42 \%)$ of respondents were of the opinion that female TB patients conformed better to treatment than males and a similar number thought that both of them equally conformed to treatment. Only male respondents felt that male TB patients conformed better to TB treatment.

Table 3: Number of respondents (\%) on their response to treatment seeking before reporting to heath facilities

\begin{tabular}{|c|c|c|c|c|c|c|c|c|c|c|}
\hline \multirow[b]{2}{*}{ Response } & \multicolumn{2}{|c|}{$1^{\text {st }}$ visit } & \multicolumn{2}{|c|}{$2^{\text {nd }}$ visit } & \multicolumn{2}{|c|}{$3^{r d}$ visit } & \multicolumn{2}{|c|}{$4^{\text {th }}$ visit } & \multicolumn{2}{|c|}{ Overall $(n=69)$} \\
\hline & No. & $\%$ & No. & $\%$ & No. & $\%$ & No. & $\%$ & No. & $\%$ \\
\hline The same health facility & 19 & 28.8 & 10 & 17.2 & 19 & 40.4 & 6 & 26.1 & 54 & 78.3 \\
\hline \multicolumn{11}{|l|}{ Public Dispensary or Health } \\
\hline Centre & 19 & 28.8 & 24 & 41.4 & 16 & 34.0 & 8 & 34.8 & 67 & 97.1 \\
\hline District hospital & 9 & 13.6 & 10 & 17.2 & 6 & 12.8 & 4 & 17.4 & 29 & 42.0 \\
\hline $\begin{array}{l}\text { Private dispensary/Health } \\
\text { Centre }\end{array}$ & 8 & 12.1 & 3 & 5.2 & - & - & 1 & 4.3 & 12 & 17.4 \\
\hline Pharmacy/Shops & 7 & 10.6 & 2 & 3.4 & - & - & & & 9 & 13.0 \\
\hline Muhimbili National Hospital & 2 & 3.0 & 9 & 15.5 & - & - & 4 & 17.4 & & \\
\hline Traditional practitioners & 2 & 3.0 & - & - & 6 & 12.8 & - & - & 8 & 11.6 \\
\hline Total & 66 & & 58 & & 47 & & 23 & & & \\
\hline
\end{tabular}




\section{Discussion}

The findings show that the majority of the TB patients were heads of households, who were bread winners for their dependants. Although this indicates that, the disease has great impact on the daily life of the families, there might be an impression of differences in health seeking behaviour and access to health services in this group than others. However, it may also be due to the effects of gender differences in social and economic status and activities performed by the individuals, which may lead to differential exposure to the TB infection (Hudelson, 1996; Garcia-Garcia et al., 2006; Ahsan et al., 2004).

The median delay of 1.5 months to seek care from health facilities corresponds with previous findings from The Gambia (Eastwood \& Hill, 2004) and Nepal (Yamasaki-Nakagawa, 2001). Several studies have documented on delays in seeking care among male and female TB patients (Wandwalo \& Morkve, 2000; Farah et al., 2006; Ahsan et al., 2004). This study found that gender variations did not affect utilisation of services. Patients had complex patterns of care seeking that contributed to the reported delay including formal health facilities and consulting traditional practitioners. The reported pattern corresponds with findings from other studies (Eastwood \& Hill, 2004; Sanou et al., 2004; Ahsan et al., 2004). Contrary to the findings by the above authors, in our study, the cost for travelling to health facilities incurred by patients to DOTS centres was perceived to be relatively low.

Although our data was not adequate to provide a significant variation between the education levels, the fact that most of the study patients had an equivalent or less than the primary school education, reflects that TB is a disease of illiterate and poor communities. Similar findings were reported in Gambia (Eastwood \& Hill, 2004). These findings may have some unpleasant social consequences such as cultivation of stigma to the disease (Johansson et al., 2000; Farah et al., 2006). Our study reports that males had little knowledge on transmission of TB than females in addition, $40 \%$ of the TB patients had no correct knowledge or were not aware of how long it takes to complete TB treatment, this findings raises a great concern over the impact of health education programmes on TB control. The variations on knowledge of the different basics of TB are not unique to the TB patients in this study. Similar differences have been reported in other studies (Fair et al., 1997; Yamasaki-Nakagawa, 2001; Sanou et al., 2004). This also explains the value of providing health education to TB patients at the clinic during the TB treatment period.
In this study, behavioural-dependent factors like HIV/AIDS epidemic and poor compliance to treatment were among the perceived causes for the increase of TB. This was further explained by the knowledge that the disease was curable depending on patient compliance to treatment and continued reliable availability of drugs. The level of satisfaction from the services provided to TB patients by health workers indicates a good performance of the different levels of the TB control programme in the country. It could be well attributed by the ongoing decentralization of DOTS services in the study region. In Tanzania, the decentralisation of TB services through DOTS clinics enables the service to reach even special groups such as prisoners, refugee's camps and other disadvantaged populations. Good patient-provider relationship and availability of TB drugs at DOTS centres are the most important factor that influences patients' compliance (Irani et al, 2007; Johansson et al., 1996). Higher compliance among females is likely to be related to the fact they are more responsible with their families than males. As an evidence of fair treatment regardless of gender, there was no difference in terms of satisfaction with services between male and female patients in general. In a study conducted in Bangladesh (Ahsan et al., 2004) most female patients were not satisfied with their health providers' behaviours, which interfered significantly with their treatment seeking behaviour.

The findings from this study have shown the experiences and perceptions of TB patients from gender perspectives, in terms of treatment seeking, behaviours associated with socio-cultural and economic barriers. Our findings also show that traditional practitioners and drug sellers, to some extent were consulted for treatment during the onset of TB symptoms. On one hand, these might sound as contributing to delays among TB patients to seek early diagnosis and treatment at health facilities. However, on the other hand, the results suggest a need to further explore how these options could potentially be used to enhance early seeking of appropriate services among TB patients. Strategies in the control of TB and other diseases should focus on advocacy, challenging not only males and females as change agents in seeking appropriate care, but also the common first contacts which include traditional healers and drug sellers.

Received 17 August 2007

Revised 11 March 2008

Accepted 13 March 2008 


\section{References}

Ahsan, G., Ahmed J., Singhasivanon, P., Kaewkungwal, J., Okanurak, K., Suwannapong, N., Akarasewi ,P., Majid, M.A., Begum, V. \& Belayetali, K. (2004) Gender difference in treatment seeking behaviours of tuberculosis cases in rural communities of Bangladesh. Southeast Asian Journal of Tropical Medicine and Public Health 35, 126-135.

Begum, V., de Colombani, P., Das Gupta, S., Salim, A.H., Hussain, H., Pietroni, M., Rahman, S., Pahan, D. \& Borgdorff, M.W. (2001) Tuberculosis and patient gender in Bangladesh: sex differences in diagnosis and treatment outcome. International Journal of Tuberculosis and Lung Disease 5, 604-610.

Eastwood, S.V. \& Hill, P.C. (2004) A gender-focused qualitative study of barriers to accessing tuberculosis treatment in The Gambia, West Africa. International Journal of Tuberculosis and Lung Disease 8, 70-75.

Fair, E., Islam, A.M. \& Chowdhury, A.S. (1997) Tuberculosis and Gender: Treatment Seeking Behaviour and Social Beliefs of Women with Tuberculosis in Rural Bangladesh. Working Paper Number 1, BRAC, Bangladesh.

Farah, M.G., Rygh, J.H, Steen, T.W, Selmer, R., Heldal, E. \& Bjune, G. (2006) Patient and health care system delays in the start of tuberculosis treatment in Norway. BMC Infectious Diseases 6, 33. doi: 10.1186/1471-2334-6-33.

Garcia-Garcia, L., DeRiemer, K., Ferreyra-Reyes, L., Bobadilla-del-Valle, M., Cano-Arellano, B., Canizales-Quintero, S., Martinez-Gamboa, A., Small, P.M., Sifuentes-Osornio, J., \& Ponce-de-Leon, A. (2006) Gender differentials of pulmonary tuberculosis transmission and reactivation in an endemic area. Thorax 61, 348-353.

Hudelson, P. (1996) Gender differentials in tuberculosis: the role of socio-economic and cultural factors. Tuberculosis and Lung Disease 77, 391-400.

Irani, L., Kabalimu, T.K. \& Kasesela, S. (2007) Knowledge and healthcare seeking behaviour of pulmonary tuberculosis patients attending Ilala District Hospital, Tanzania. Tanzania Health Research Bulletin 9, 169-173.

Johansson, E., Diwan, V.K., Huong, N.D. \& Ahlberg, B.M. (1996) Staff and patient attitudes to tuberculosis and compliance with treatment: An exploratory study in a district in Vietnam. Tubercle and Lung Disease 77, 178-183.
Johansson, E., Long, N.H., Diwan, V.K. \& Winkvist, A. (2000) Gender and tuberculosis control: perspectives on health seeking behaviour among men and women in Vietnam. Health Policy 52, 33-51.

Lorenz, N. \& Mtasiwa, D. (2004) Health in the Urban Environment: Experience from Dar es Salaam, Tanzania. Annals of the New York Academy of Sciences 1023, 159-163.

Mangesho, P.E., Shayo, E., Makunde, W.H., Keto, G.B.S., Mandara, C.I., Kamugisha, M.L., Kilale, A.M. \& Ishengoma, D.R.S. (2007) Community knowledge, attitudes and practices towards tuberculosis and its treatment in Mpwapwa District, central Tanzania. Tanzania Health Research Bulletin 9, 38-43.

Okeibunor, J.C., Onyeneho, N.G., Chukwu, J.N. \& Post, E. (2007) Where do tuberculosis patients go for treatment before reporting to DOTS clinics southern Nigeria. Tanzania Health Research Bulletin 9, 94-101.

Okeke, T.A. \& Aguwa, E.N. (2006) Evaluation of the implementation of directly observed treatment short course by private medical practitioners in the management of tuberculosis in Enugu, Nigeria. Tanzania Health Research Bulletin 8, 86-89.

Sanou, A., Dembele, M., Theobald, S. \& Macq, J. (2004) Access and adhering to tuberculosis treatment: barriers faced by patients and communities in Burkina Faso. International Journal of Tuberculosis and Lung Disease 8, 1479-1483.

Thorson, A. \& Diwan, V.K. (2001) Gender inequalities in tuberculosis: aspects of infection, notification rates, and compliance Current Opinion in Pulmonary Medicine 7, 165-169.

Wandwalo, E.R. \& Morkve, O. (2000) Delay in tuberculosis cases finding and treatment in Mwanza, Tanzania. International Journal of Tuberculosis and Lung Disease 4, 133-138.

WHO (2002) An Expanded DOTS Framework for Effective Tuberculosis Control. World Health Organization, Geneva

WHO (2005) Tuberculosis: The Global Burden. http://www.who.int/tb/publications/tb global facts sep05

Yamasaki-Nakagawa, M. (2001) Gender difference in delays to diagnosis and health care seeking behaviour in a rural area of Nepal. International Journal of Tuberculosis and Lung Disease 5, 24-31.

Zhang, T., Liu, X. Bromley, H. \& Tang, S. (2007) Perceptions of tuberculosis and health seeking behaviour in rural Inner Mongolia, China. Health Policy 81, 155-165. 